\title{
ELLIPTIC SURFACES WITHOUT 1-HANDLES
}

\author{
KOUICHI YASUI
}

\begin{abstract}
Harer-Kas-Kirby conjectured that every handle decomposition of the elliptic surface $E(1)_{2,3}$ requires both 1- and 3-handles. We prove that the elliptic surface $E(n)_{p, q}$ has a handle decomposition without 1-handles for $n \geq 1$ and $(p, q)=(2,3),(2,5),(3,4),(4,5)$.
\end{abstract}

\section{INTRODUCTION}

It is not known whether or not the 4 -sphere $S^{4}$ and the complex projective plane $\mathbf{C P}^{2}$ admit an exotic smooth structure. If such a structure exists, then each handle decomposition of it has at least either a 1- or 3-handle (cf. 8 ). On the contrary, many simply connected closed topological 4-manifolds are known to admit infinitely many different smooth structures which have neither 1- nor 3-handles in their handle decompositions (cf. Gompf-Stipsicz [4]).

Problem 4.18 in Kirby's problem list [6] is the following: "Does every simply connected, closed 4-manifold have a handlebody decomposition without 1-handles? Without 1- and 3-handles?" It is not known whether or not the simply connected elliptic surface $E(n)_{p, q}(n \geq 1, p, q \geq 2, \operatorname{gcd}(p, q)=1)$ admits a handle decomposition without 1-handles. In particular, Harer, Kas and Kirby conjectured in [5] that every handle decomposition of $E(1)_{2,3}$ requires both 1- and 3-handles. Gompf [3] notes the following: it is a good conjecture that $E(n)_{p, q}(p, q \geq 2)$ has no handle decomposition without 1 - and 3-handles.

In [7] and [8] we constructed a homotopy $E(1)_{2,3}$ which has the same SeibergWitten invariant as $E(1)_{2,3}$ and has a handle decomposition without 1- and 3handles. Recently Akbulut [1] proved that $E(1)_{2,3}$ has a handle decomposition without 1- and 3-handles, by using knot surgery on $E(1)$ and investigating a dual handle decomposition. He also proved that infinitely many different smooth structures on $\mathbf{C P}^{2} \# 9 \overline{\mathbf{C P}^{2}}$ admit handle decompositions without 1-handles.

In this paper, we prove the theorem below by improving our previous procedure ([7, [8]). Our method is different from Akbulut.

Theorem 1.1. The elliptic surface $E(n)_{p, q}$ has a handle decomposition without 1 -handles, for $n \geq 1$ and $(p, q)=(2,3),(2,5),(3,4),(4,5)$.

Acknowledgement . The author wishes to express his deep gratitude to his adviser, Hisaaki Endo, for encouragements and helpful comments. The author would like to thank Selman Akbulut, Motoo Tange and Yuichi Yamada for discussions. This work was partially done during the author's stay at Michigan State University.

Date: February 22, 2008. Revised on July 6, 2008.

2000 Mathematics Subject Classification. Primary 57R55, Secondary 57R65, 57N13.

Key words and phrases. 4-manifold; 1-handle; elliptic surface.

The author is partially supported by JSPS Research Fellowships for Young Scientists. 
The author is grateful for their hospitality. Finally, the author would like to thank the referee for his/her useful suggestion (see the proof of Proposition 3.3).

\section{RATIONAL BLOW-DOWN}

In this section we review the rational blow-down introduced by Fintushel-Stern 2]. See also Gompf-Stipsicz [4].

Let $C_{p}$ and $B_{p}(p \geq 2)$ be the smooth 4-manifolds defined by Kirby diagrams in Figure 1. The boundary $\partial C_{p}$ of $C_{p}$ is diffeomorphic to the lens space $L\left(p^{2}, p-1\right)$ and to the boundary $\partial B_{p}$ of $B_{p}$.

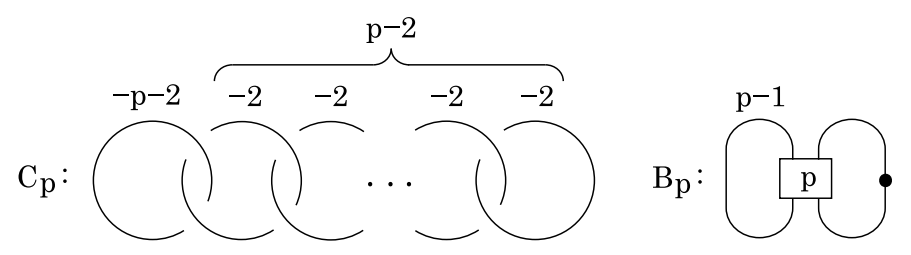

FIGURE 1.

Suppose that $C_{p}$ embeds in a smooth 4-manifold $X$. Let $X_{(p)}$ be a smooth 4manifold obtained from $X$ by removing $C_{p}$ and gluing $B_{p}$. The 4-manifold $X_{(p)}$ is called the rational blow-down of $X$ along $C_{p}$. Note that $X_{(p)}$ is uniquely determined up to diffeomorphism by a fixed pair $\left(X, C_{p}\right)$. This operation has the following relation with the logarithmic transformation.

Theorem 2.1 (Fintushel-Stern [2], see also Gompf-Stipsicz [4]). Suppose that a smooth 4-manifold $X$ contains a cusp neighborhood, that is, a 0-handle with a 2 handle attached along a 0 -framed right trefoil knot. Let $X_{p}$ be the smooth 4-manifold obtained from $X$ by performing a logarithmic transformation of multiplicity $p$ in the cusp neighborhood. Then there exists a copy of $C_{p}$ in $X \#(p-1) \overline{\mathbf{C P}^{2}}$ such that the rational blow-down of $X \#(p-1) \overline{\mathbf{C P}^{2}}$ along the copy of $C_{p}$ is diffeomorphic to $X_{p}$.

\section{Proof}

In this section we prove Theorem 1.1. We do not draw (whole) Kirby diagrams of elliptic surfaces. However, one can draw whole diagrams of elliptic surfaces without 1-handles, following the procedures in this section.

Let $E(n)$ be the simply connected elliptic surface with Euler characteristic $12 n$ and with no multiple fibers, and $E(n)_{p_{1}, \ldots, p_{k}}$ the elliptic surface obtained from $E(n)$ by performing logarithmic transformations of multiplicities $p_{1}, \ldots, p_{k}$.

Proposition 3.1. For $n \geq 1$, the elliptic surface $E(n)_{2}$ has handle decompositions as in Figure 2 and 3 . Each obvious cusp neighborhood in Figure 2 and 3 is isotopic to a regular neighborhood of a cusp fiber of $E(n)_{2}$.

Proof. $E(n)_{p}$ admits a handle decomposition in Figure 9 (see Gompf-Stipsicz [4, page $315 \sim 316$ ] and Harer-Kas-Kirby [5]). The obvious cusp neighborhood in Figure 9 is isotopic to a regular neighborhood of a cusp fiber of $E(n)_{p}$ (see [4] and [5]). Figure 10 is the $p=2$ case of Figure 9. Note that we do not draw $6 n-1$ 2-handles in Figure 10. We change Figure 10 into Figure 2 and 3 without sliding the cusp neighborhood over any handles, as follows. In Figure 10, we slide $-4 n+2$ 


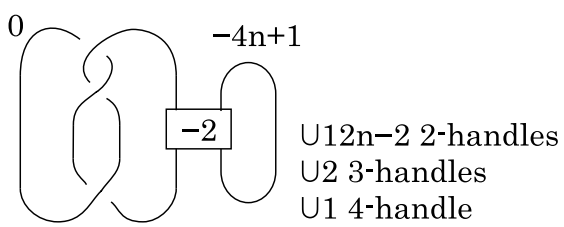

Figure 2. $E(n)_{2}$

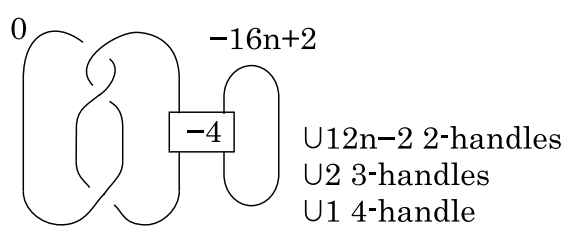

FiguRE 3. $E(n)_{2}$

framed knot over vertical -1 framed knots as shown in Figure 10 13 . Note that $\frac{k}{2}$ in the boxes denotes $k$ right half-twists. By repeating handle slides similar to Figure 11 13, we obtain Figure 14. An isotopy gives Figure 15. By canceling 1-handles, we get Figure 2,

In Figure 15] we slide a vertical -1 framed knot over $-4 n+1$ framed knot. We get Figure 16. We slide $-4 n$ framed knot over $-4 n+1$ framed knot as shown in Figure 17. Sliding $-16 n+3$ framed knot over a vertical -1 framed knot gives Figure 18. By repeating handle slides similar to Figure 11] 13, we obtain Figure 19 An isotopy gives Figure 20. By canceling 1-handles, we get Figure 3 .

Proposition 3.2. For $n \geq 1$, the elliptic surface $E(n)_{3}$ admits a handle decomposition as in Figure 4. The obvious cusp neighborhood in Figure 4 is isotopic to a regular neighborhood of a cusp fiber of $E(n)_{3}$.

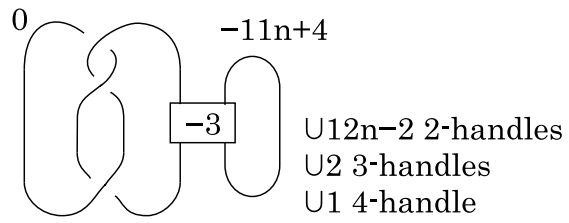

FiguRE $4 . E(n)_{3}$

Proof. Figure 21 is the $p=3$ case of Figure 9. We change Figure 21 into Figure 4 without sliding the cusp neighborhood over any handles. In Figure 21, we slide $-9 n+3$ framed knot over vertical -1 framed knots as shown in Figure 21 28. An isotopy gives Figure 29. We get Figure 30 by sliding $-9 n+2$ framed knot over a vertical -1 framed knot. In the $n \geq 2$ case, we obtain Figure 31 by repeating handle slides similar to Figure 21] 30. We get the $n=1$ case of Figure 31 by an isotopy in the $n=1$ case of Figure 21. Handle slides similar to Figure 21 24 give Figure 32. An isotopy gives Figure 33. By canceling 1-handles, we get Figure4.

Proposition 3.3. For $n \geq 1$, the elliptic surface $E(n)_{4}$ admits a handle decomposition as in Figure 5 . The obvious cusp neighborhood in Figure 5 is isotopic to a regular neighborhood of a cusp fiber of $E(n)_{4}$.

Proof. In Figure 9 of $E(n)_{p}$, we repeat handle slides shown in Figure 34, We then get the diagram of $E(n)_{p}$ in Figure 35. (This diagram is a key of our proof for $n \geq 2$. The way to construct this diagram is suggested by the referee.) Note that we did not slide the cusp neighborhood in Figure 9 over any handles. Figure 36 is 


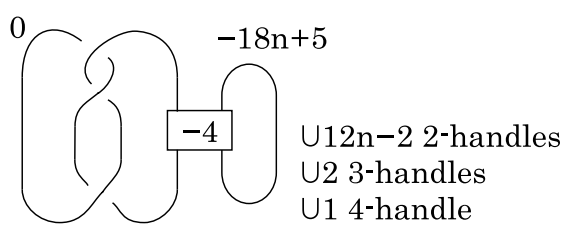

Figure 5. $E(n)_{4}$

the $p=4$ case of Figure 35. We change Figure 36 into Figure 5 without sliding the cusp neighborhood over any handles, as follows.

The $n \geq 2$ case. We slide handles as shown in Figure 36 $\sim 46$. We then get Figure 47. Isotopies give Figure 48 and 49. We have Figure 53 by handle slide as shown in Figure $49 \sim 52$ By repeating handle slides similar to Figure $36 \sim 53$. we obtain Figure 54. We slide handles similarly to Figure 36 433 , We then get Figure 55. An isotopy gives Figure 56. By cancelling 1-handles, we have Figure 5 ,

The $n=1$ case. Figure 54 is isotopic to the $n=1$ case of Figure 36. We slide handles similarly to Figure 36 $\sim 43$. We then get Figure 55. An isotopy gives Figure 56. By cancelling 1-handles, we have Figure 5.

Lemma 3.4. Suppose that a simply connected closed smooth 4-manifold $X$ has a handle decomposition as in Figure [6. Here $q$ is an arbitrary integer. $h_{2}$ and $h_{3}$ are arbitrary non-negative integers. Let $X_{(p)}$ be the rational blow-down of $X$ along the copy of $C_{p}$ in Figure 6 . Then $X_{(p)}$ admits a handle decomposition

$$
X_{(p)}=\text { one } 0 \text {-handle } \cup\left(h_{2}+1\right) \text { 2-handles } \cup h_{3} \text { 3-handles } \cup \text { one 4-handle. }
$$

In particular $X_{(p)}$ admits a handle decomposition without 1-handles.

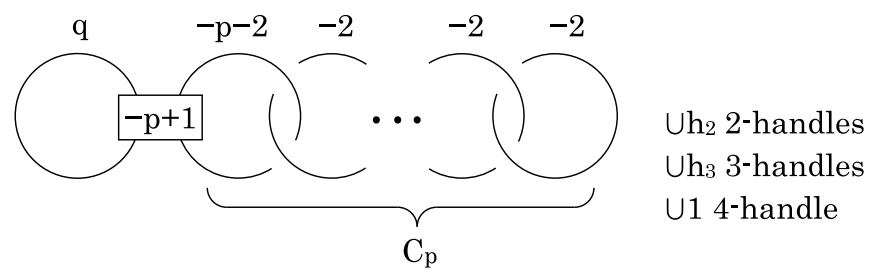

FiguRE 6. Handle decomposition of $X$

Proof. Draw a Kirby diagram of $X_{(p)}$, following the procedure introduced in GompfStipsicz [4, Section 8.5] (see also [4, page 516 Solution of Exercise 8.5.1.(a)]). Then we have a handle decomposition of $X_{(p)}$ as in Figure 7 We easily get a meridian of the unique dotted circle by a handle slide. Thus we can cancel the 1-handle/2handle pair.

Corollary 3.5. Suppose that a simply connected closed smooth 4-manifold $X$ has a handle decomposition as in Figure 8 . Here $q$ is an arbitrary integer. $h_{2}$ and $h_{3}$ are arbitrary non-negative integers. Let $X_{p}$ be the smooth 4-manifold obtained from $X$ by performing a logarithmic transformation of multiplicity $p$ in the obvious cusp neighborhood in Figure 8. Then $X_{p}$ admits a handle decomposition

$$
X_{p}=\text { one } 0 \text {-handle } \cup\left(h_{2}+2\right) \text { 2-handles } \cup h_{3} \text { 3-handles } \cup \text { one 4-handle. }
$$




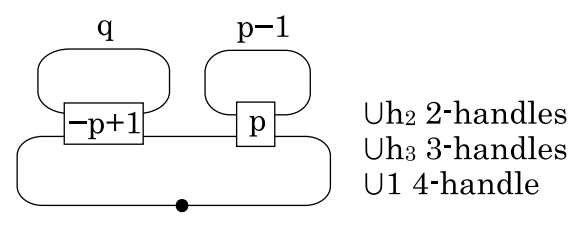

FIgURE 7. Handle decomposition of $X_{(p)}$

In particular $X_{p}$ admits a handle decomposition without 1-handles.

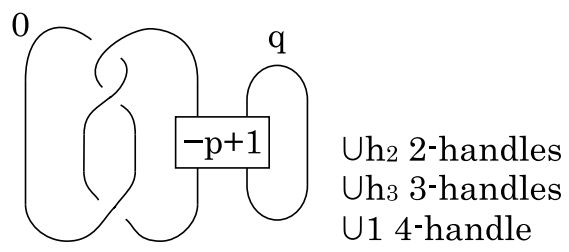

Figure 8. Handle decomposition of $X$

Proof. Construct $C_{p}$ from Figure 8, following the procedure given by FintushelStern [2, Example 1] (and Gompf-Stipsicz [4, Section 8.5]). Then we have an embedding of $C_{p}$ into $X \#(p-1) \overline{\mathbf{C P}^{2}}$ such that the rational blow-down of $X \#(p-$ 1) $\overline{\mathbf{C P}^{2}}$ along $C_{p}$ is diffeomorphic to $X_{p}$. This embedding of $C_{p}$ clearly satisfies the assumption of Lemma 3.4. Therefore we get the required handle decomposition of $X_{p}$.

Remark 3.6. One can prove Corollary 3.5 without using rational blow-downs. Follow the procedure given by Gompf [3, Section 4].

Propositions 3.1, 3.2 and 3.3 together with Corollary 3.5 clearly give the following main theorem:

Theorem 3.7. For $n \geq 1$ and $(p, q)=(2,3),(2,5),(3,4),(4,5)$, the elliptic surface $E(n)_{p, q}$ has a handle decomposition

one 0 -handle $\cup 12 n$ 2-handles $\cup$ two 3-handles $\cup$ one 4-handle.

\section{FURTHER REMARKS}

We finish this paper by making some remarks.

Remark 4.1. A key of our proof of the main theorem is to elliminate extra twists of a 2-handle of $E(n)_{p}$ so that we can apply Corollary 3.5. To carry out the key, we used many vertical -1 framed 2-handles of $E(n)_{p}$ in Figure 9 or 35. Perhaps, we may obtain more examples of elliptic surfaces without 1-handles by additionally using horizontal 2-handles of $E(n)_{p}$ in Figure 9 or 35 .

Remark 4.2. In [7] and [8, we constructed a smooth 4-manifold $E_{3}^{\prime}$ which is homeomorphic to $E(1)_{2,3}$. The 4-manifold $E_{3}^{\prime}$ has the same Seiberg-Witten invariant as $E(1)_{2,3}$ and has a handle decomposition without 1- and 3-handles. $E_{3}^{\prime}$ is constructed from $\mathbf{C P}^{2} \# 13 \overline{\mathbf{C P}^{2}}$ by rationally blowing down $C_{5}$. However, it is 
not known whether or not $E(1)_{2,3}$ can be obtained from $\mathbf{C P}^{2} \# 13 \overline{\mathbf{C P}^{2}}$ by rationally blowing down $C_{5}$. We do not know whether or not manifolds in 8 are diffeomorphic to $E(1)_{2, q}(q=3,5)$.

Remark 4.3. It seems more interesting to investigate handle decompositions of exotic 4-manifolds with small Euler characteristics, because there exist no exotic $S^{4}$ and no exotic $\mathbf{C P}^{2}$ which admit handle decompositions without 1- and 3-handles. In [9], we constructed exotic $\mathbf{C P}^{2} \# n \overline{\mathbf{C P}^{2}}(5 \leq n \leq 9)$ which admit neither 1- nor 3 -handles for $7 \leq n \leq 9$.

\section{REFERENCES}

[1] S. Akbulut, The Dolgachev Surface, arXiv:0805.1524

[2] R. Fintushel and R. J. Stern, Rational blowdowns of smooth 4-manifolds, J. Differential Geom. 46 (1997), no. 2, 181-235.

[3] R. E. Gompf, Nuclei of elliptic surfaces, Topology 30 (1991), no. 3, 479-511.

[4] R. E. Gompf and A. I. Stipsicz, 4-manifolds and Kirby calculus, Graduate Studies in Mathematics, 20. American Mathematical Society, 1999.

[5] J. Harer, A. Kas and R. Kirby, Handlebody decompositions of complex surfaces, Mem. Amer. Math. Soc. 62 (1986), no. 350.

[6] R. Kirby, Problems in low-dimensional topology, in Geometric Topology (W. Kazez ed.), AMS/IP Stud. Adv Math. vol. 2.2, Amer. Math. Soc. 1997, 35-473.

[7] K. Yasui, An exotic rational elliptic surface without 1- or 3-handles, Intelligence of low dimensional topology 2006, 375-382, Ser. Knots Everything, 40, World Sci. Publ., Hackensack, NJ, 2007.

[8] K. Yasui, Exotic rational elliptic surfaces without 1-handles, Algebr. Geom. Topol. 8 (2008), 971-996.

[9] K. Yasui, Small exotic rational surfaces without 1- and 3-handles, arXiv:0807.0373

Department of Mathematics, Graduate School of Science, Osaka University, ToyONAKA, OSAKA 560-0043, JAPAN

E-mail address: kyasui@cr.math.sci.osaka-u.ac.jp 


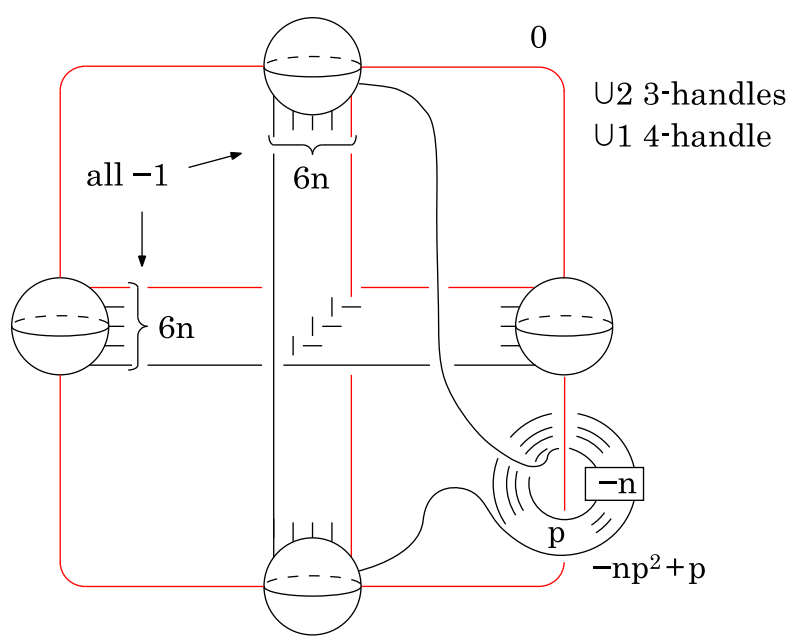

FigURE 9. $E(n)_{p}$

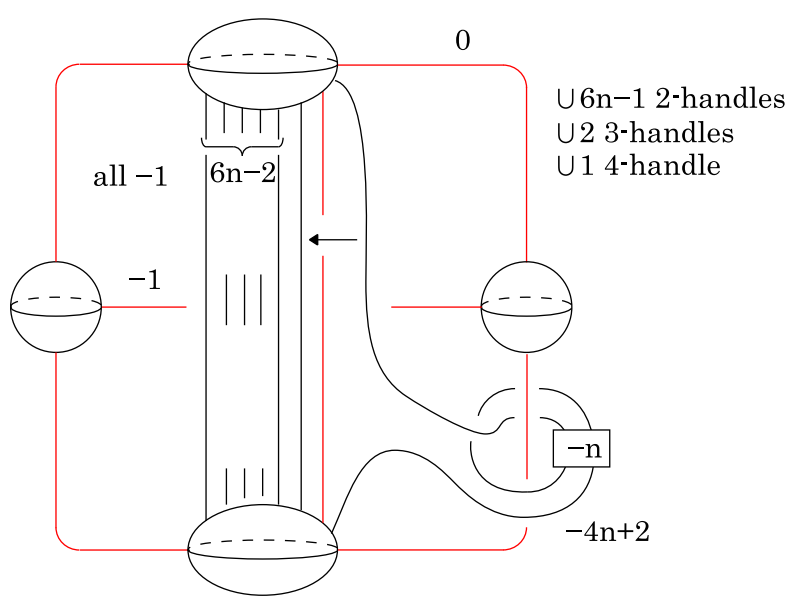

Figure $10 . \quad E(n)_{2}$

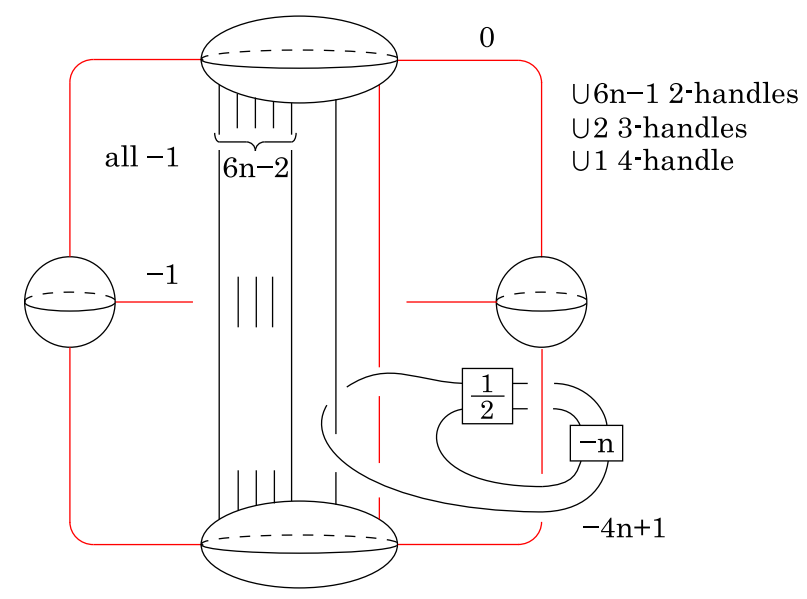

Figure $11 . E(n)_{2}$ 


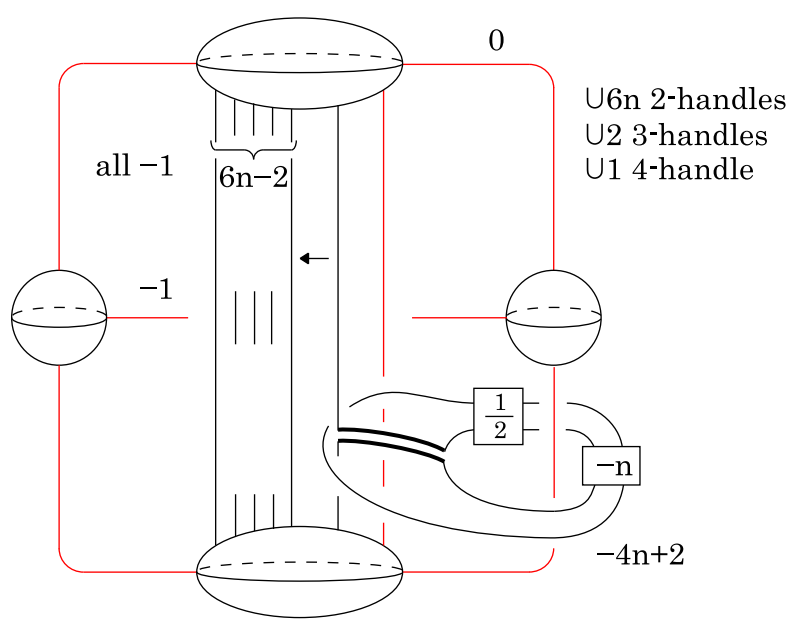

Figure $12 . \quad E(n)_{2}$

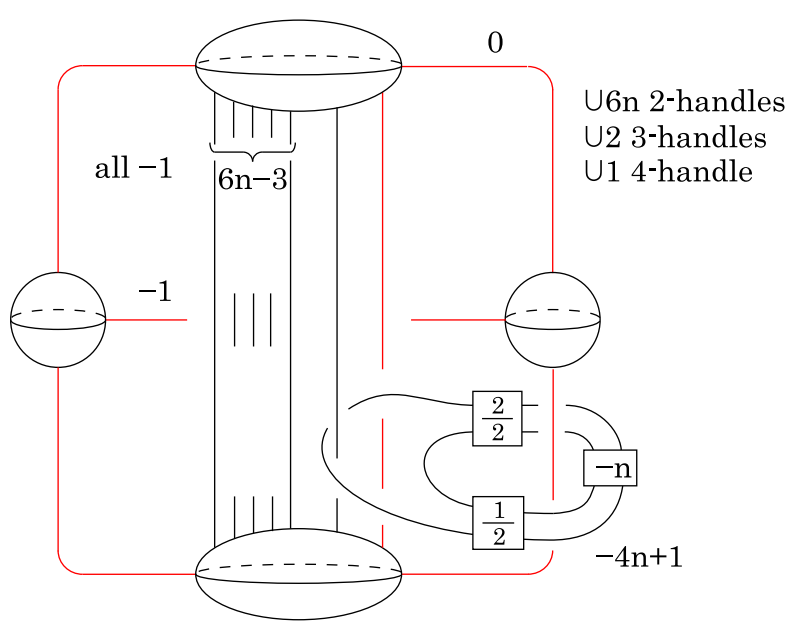

FiguRE $13 . \quad E(n)_{2}$

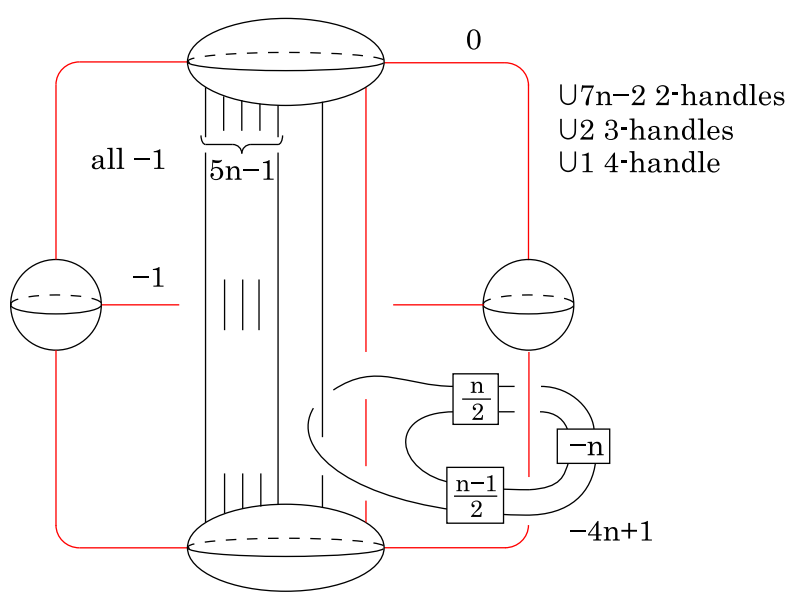

Figure 14. $E(n)_{2}$ 


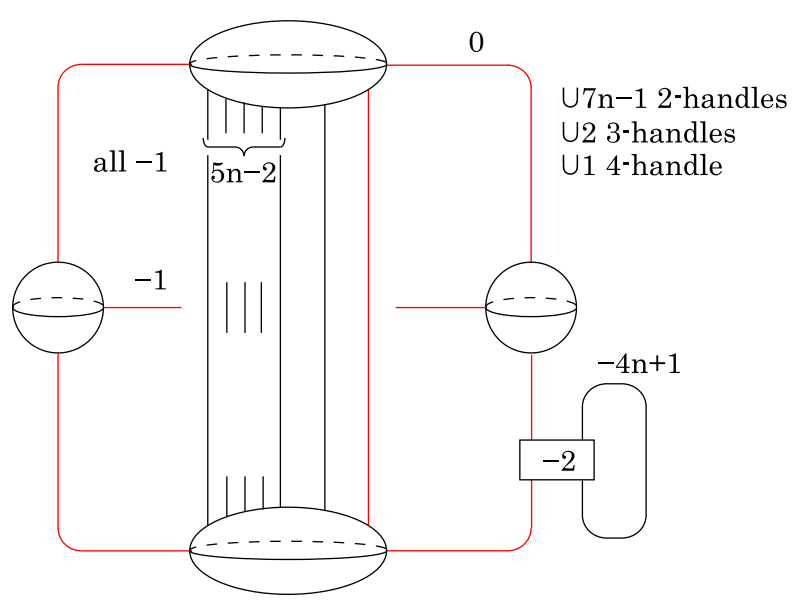

Figure $15 . \quad E(n)_{2}$

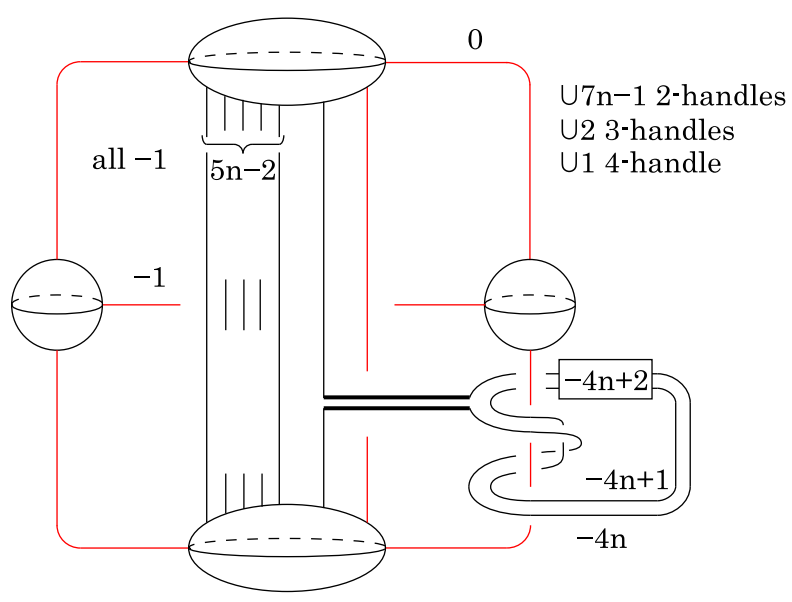

Figure $16 . \quad E(n)_{2}$

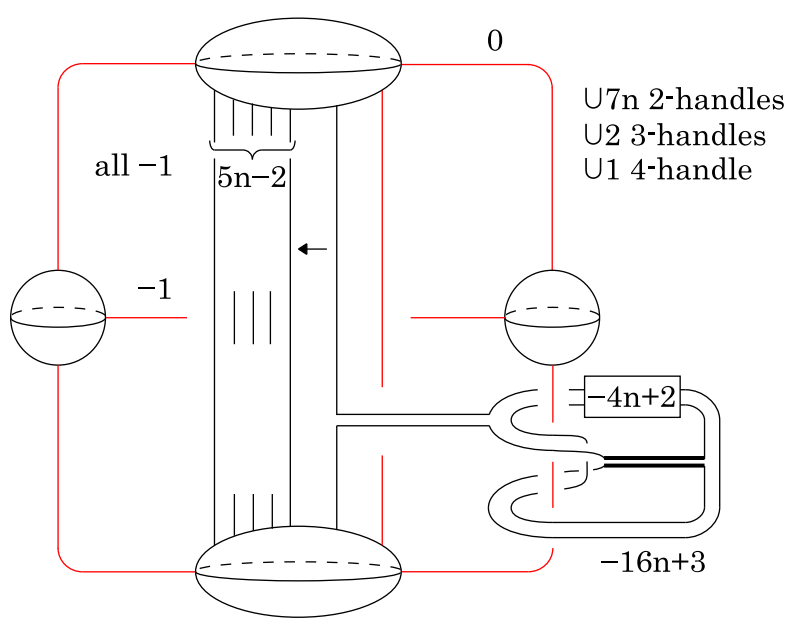

Figure 17. $E(n)_{2}$ 


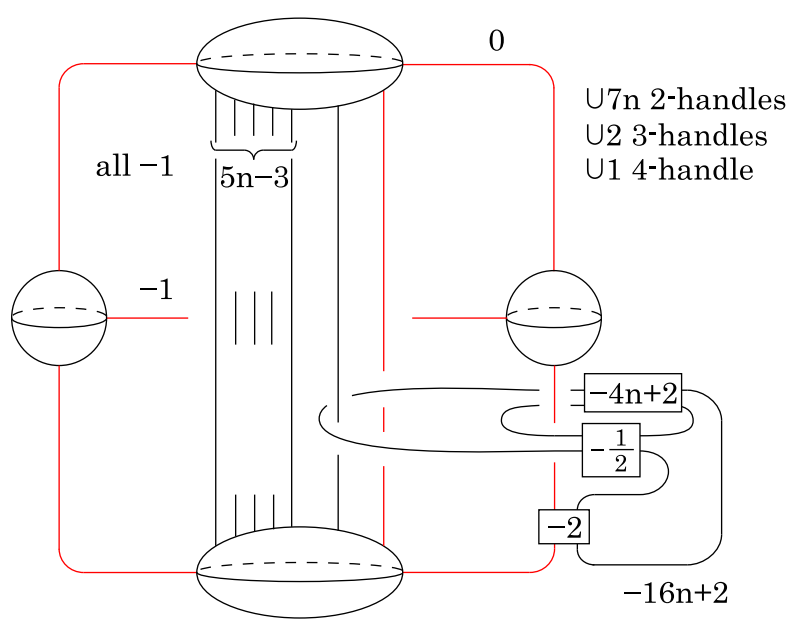

Figure $18 . \quad E(n)_{2}$

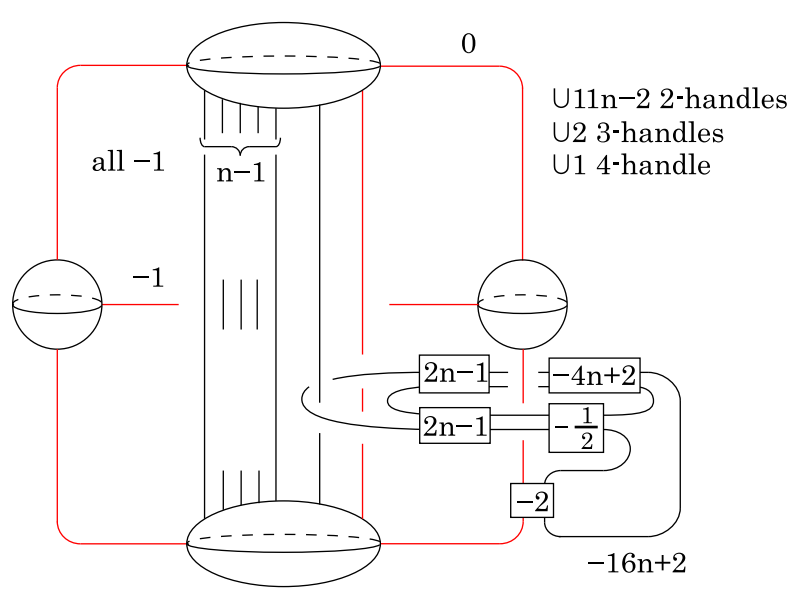

Figure 19. $E(n)_{2}$

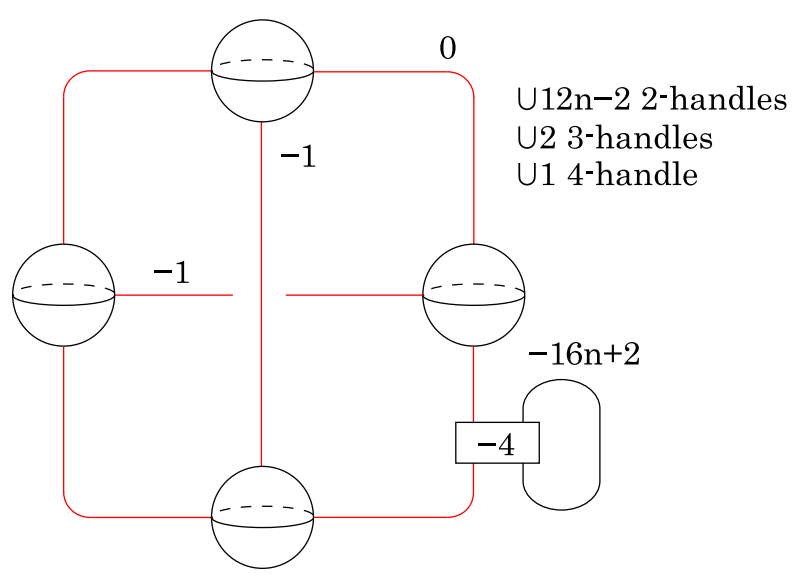

Figure 20. $E(n)_{2}$ 


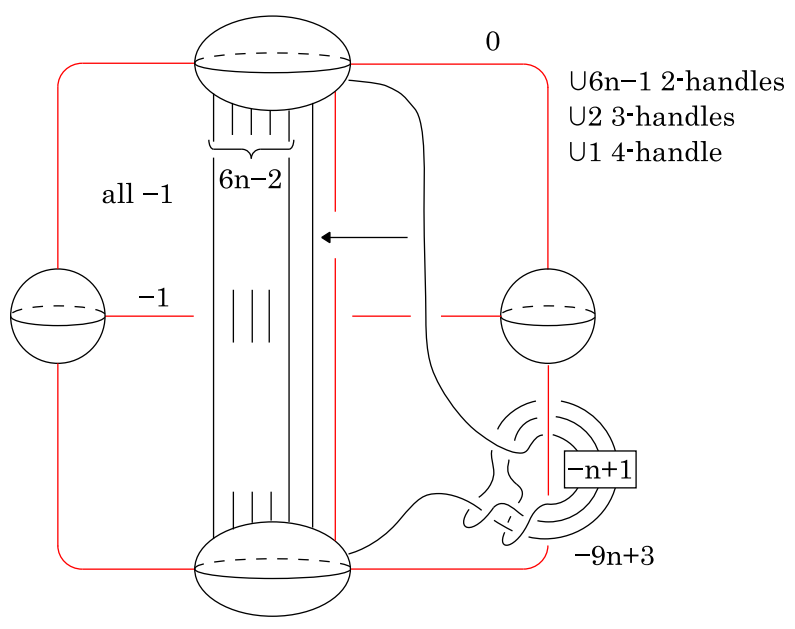

Figure $21 . E(n)_{3}$

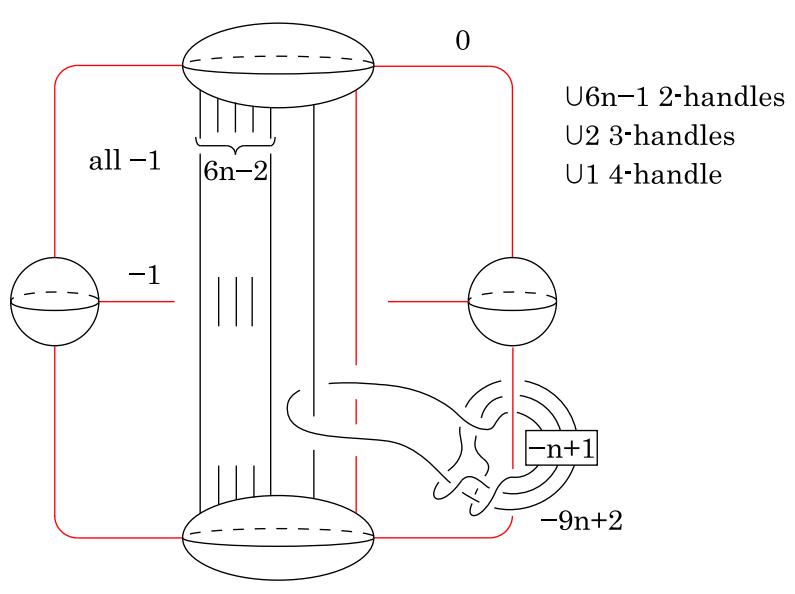

Figure 22. $E(n)_{3}$

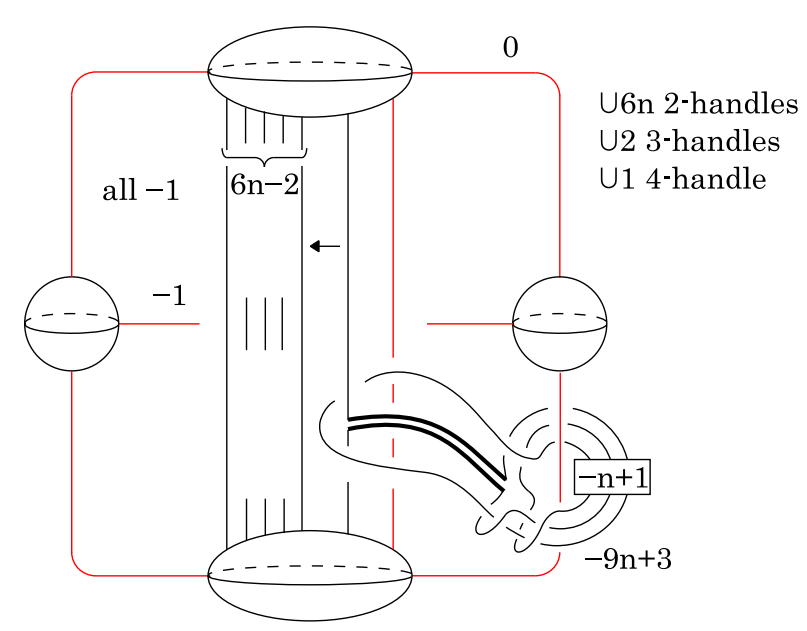

Figure 23. $E(n)_{3}$ 


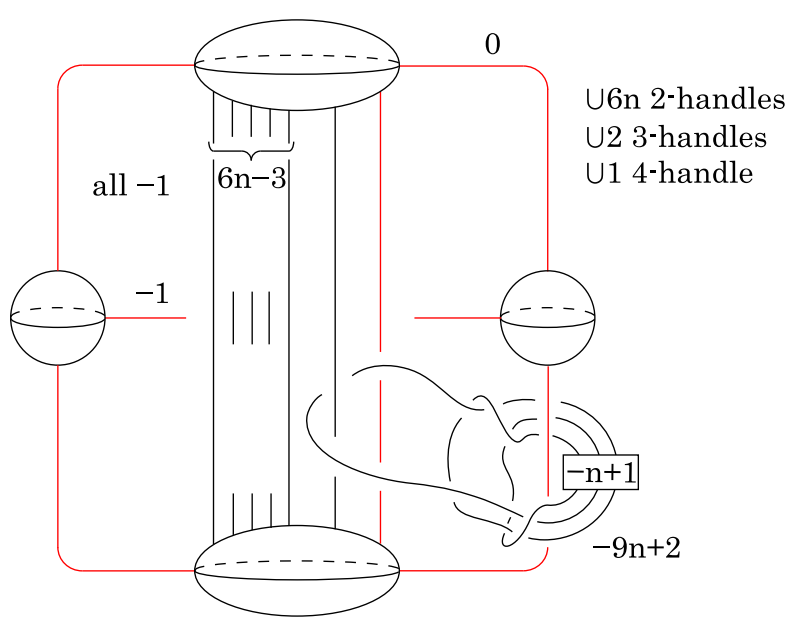

Figure 24. $E(n)_{3}$

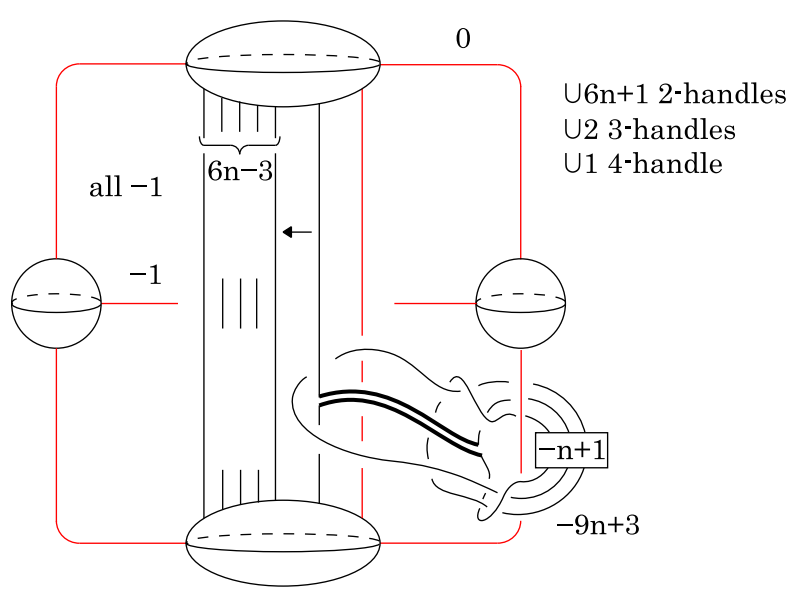

Figure 25. $E(n)_{3}$

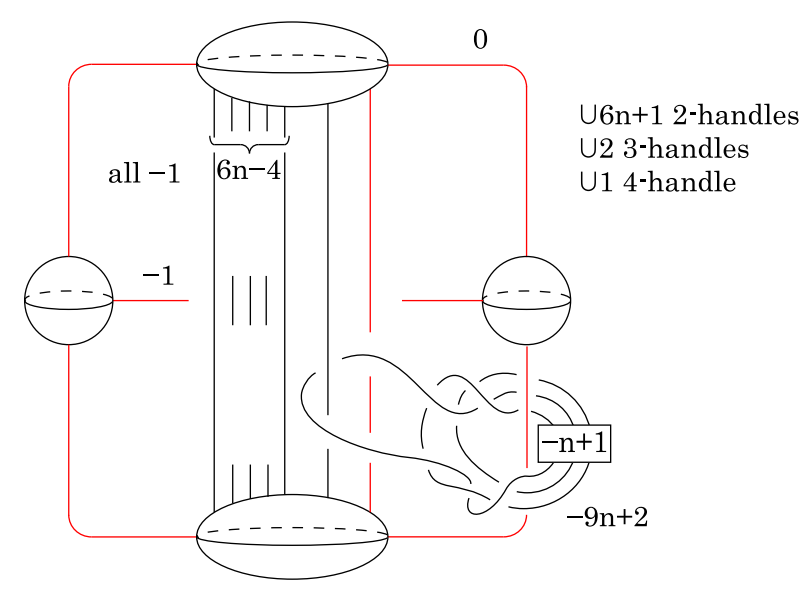

FiguRE 26. $E(n)_{3}$ 


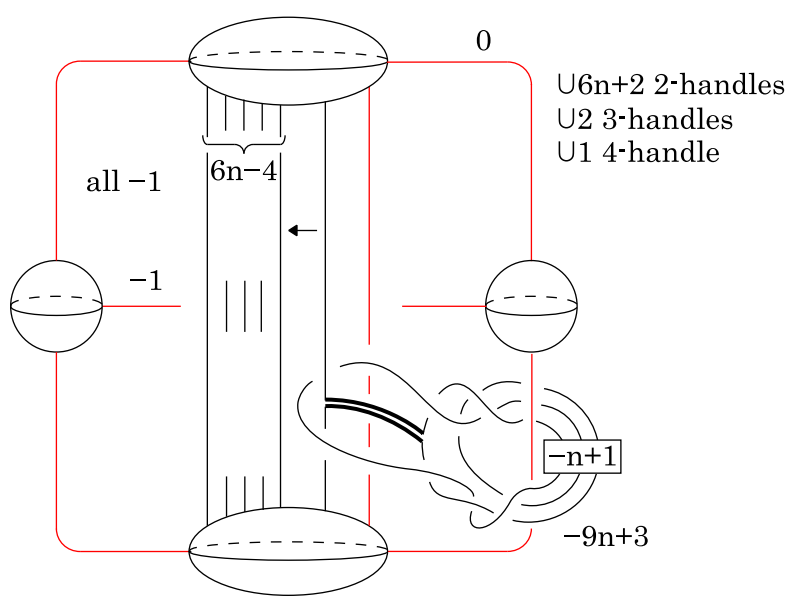

FiguRE 27. $E(n)_{3}$

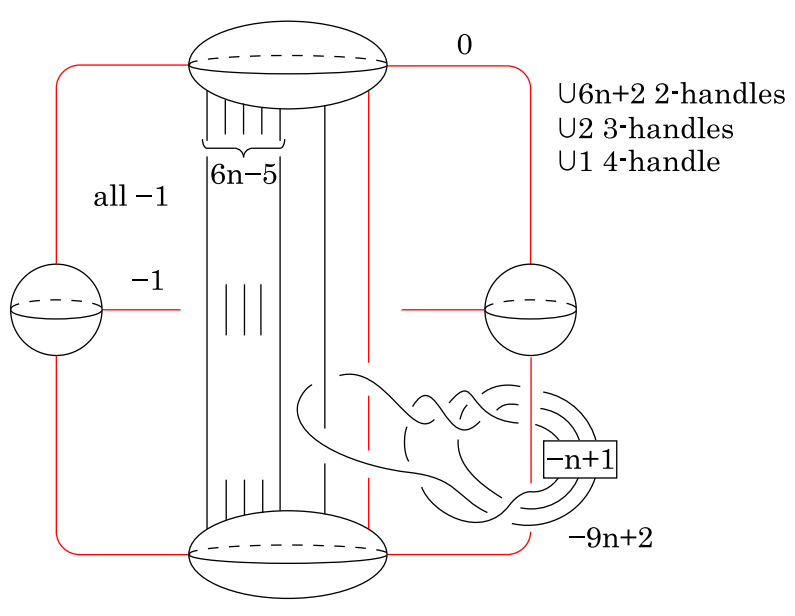

Figure 28. $E(n)_{3}$

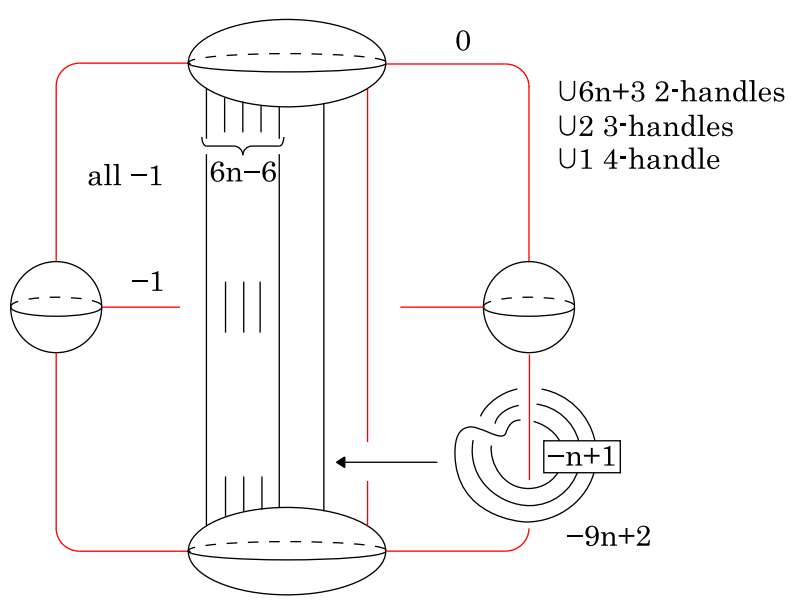

Figure 29. $E(n)_{3}$ 


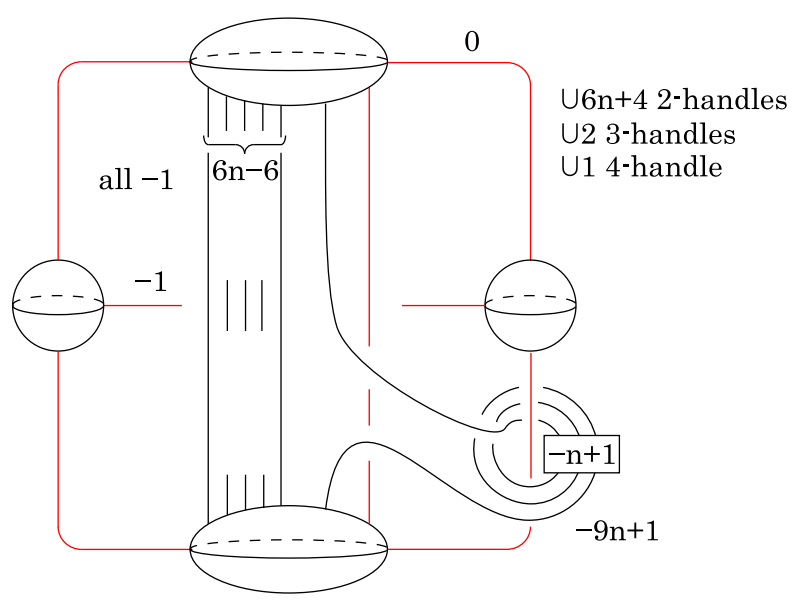

Figure $30 . \quad E(n)_{3}$

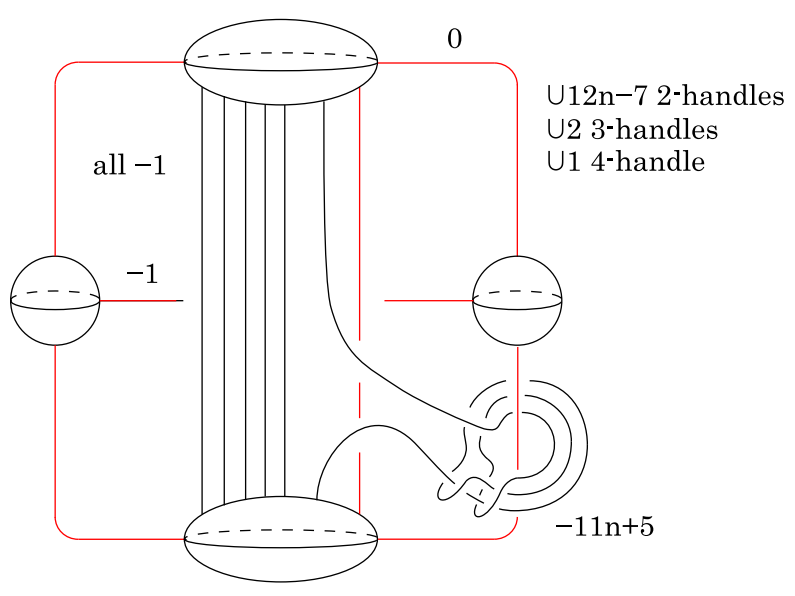

Figure $31 . E(n)_{3}$

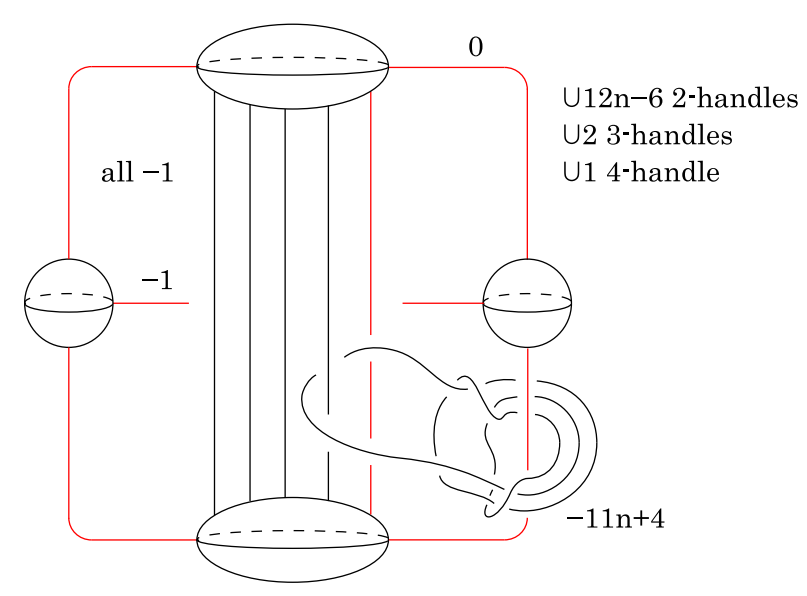

FIGURE $32 . \quad E(n)_{3}$ 


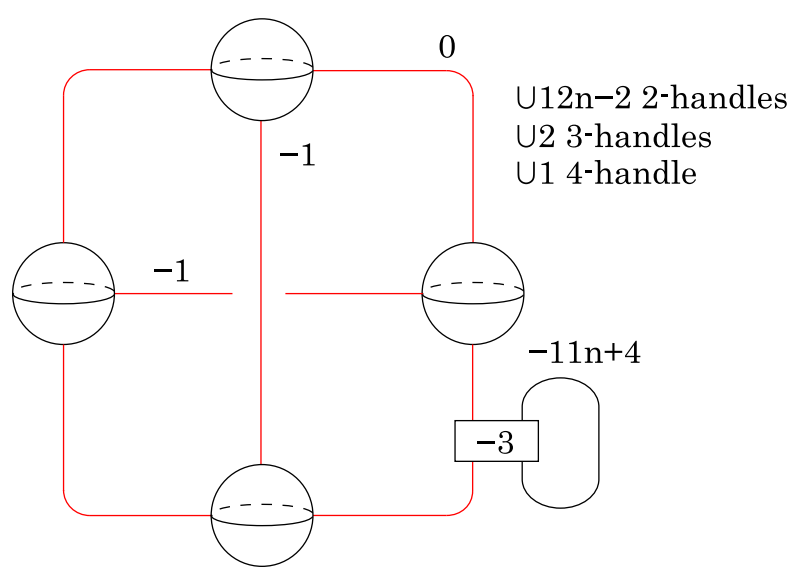

FiguRE $33 . E(n)_{3}$
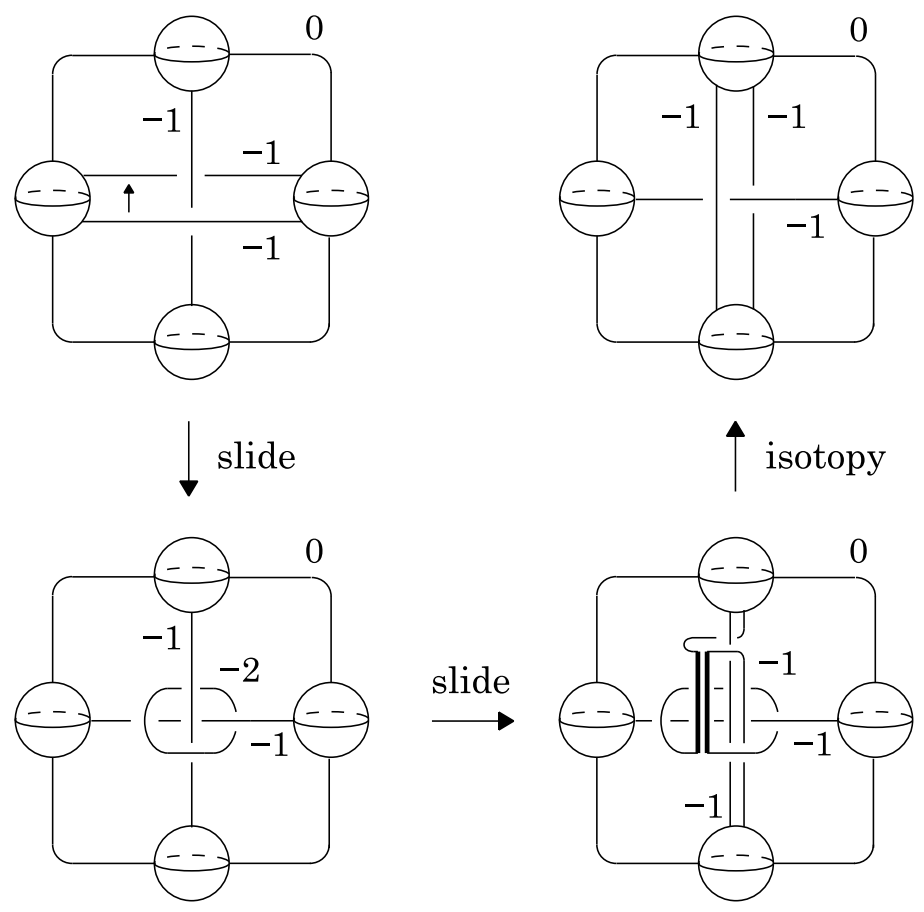

Figure 34 . handle slides 


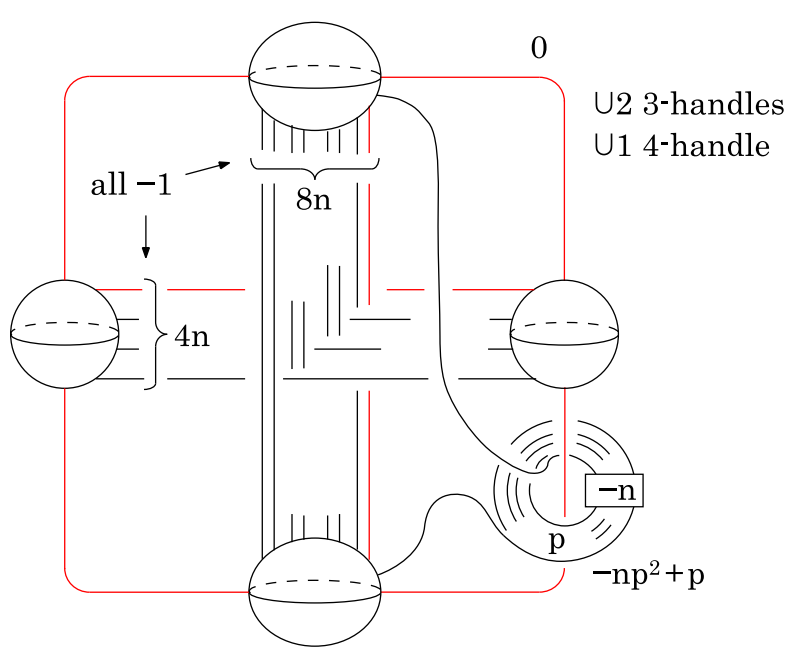

Figure $35 . E(n)_{p}$

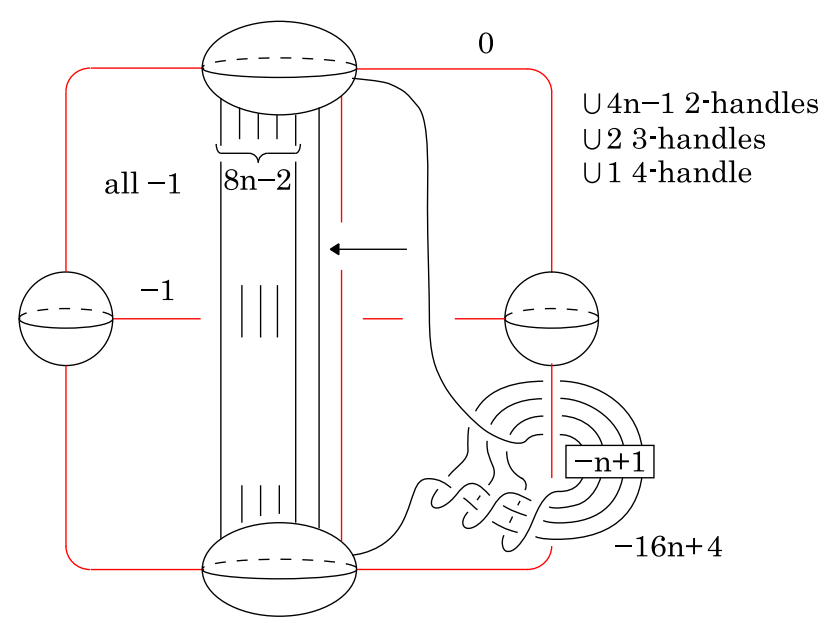

Figure $36 . \quad E(n)_{4}$

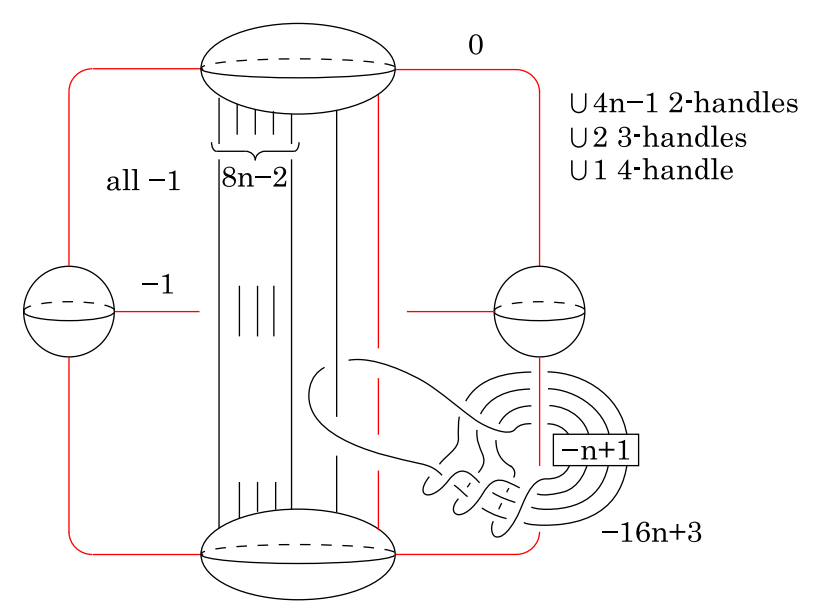

Figure $37 . E(n)_{4}$ 


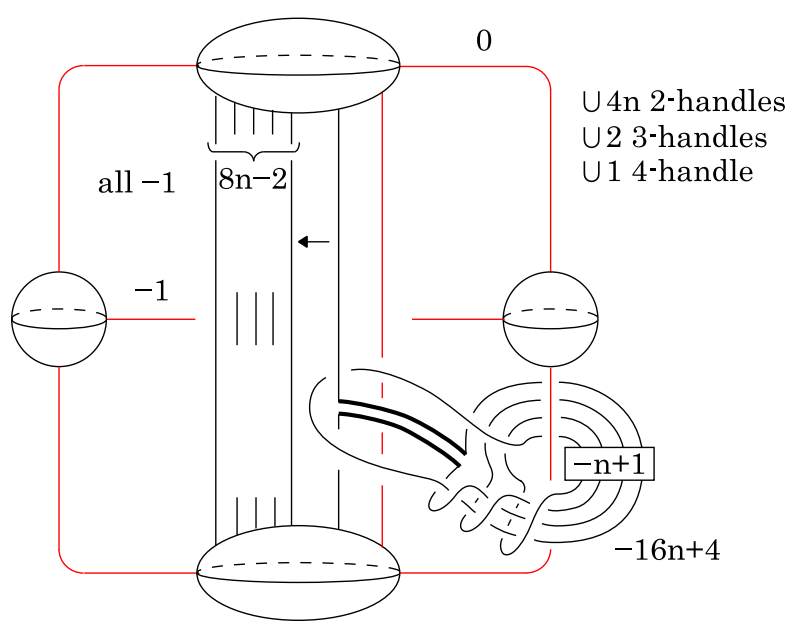

Figure $38 . \quad E(n)_{4}$

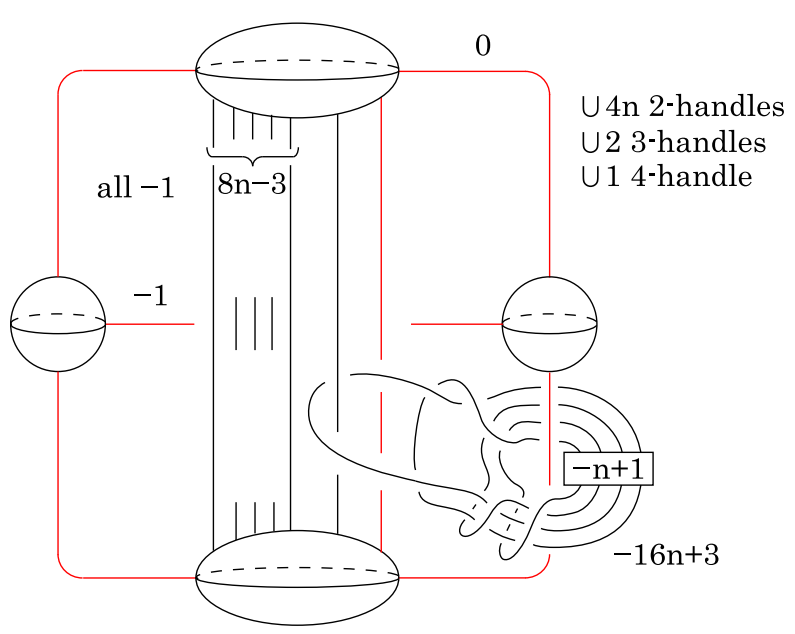

Figure $39 . \quad E(n)_{4}$

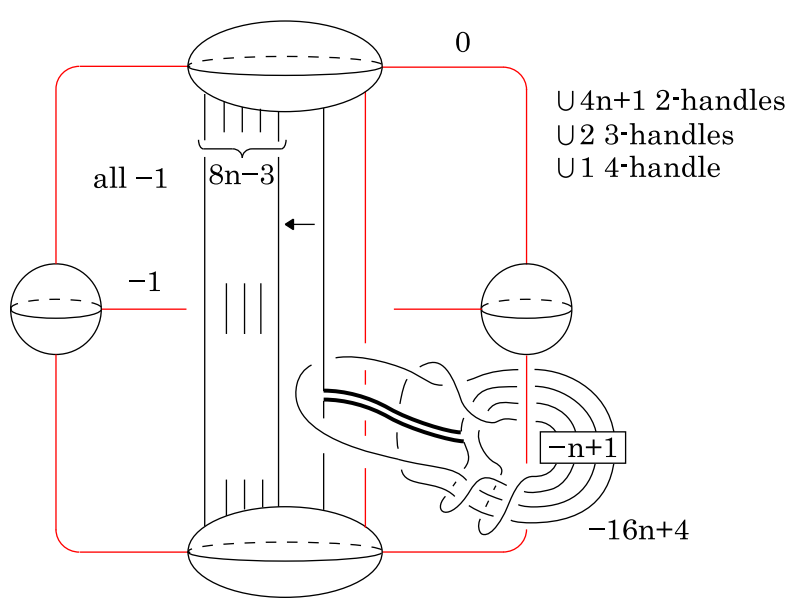

Figure $40 . \quad E(n)_{4}$ 


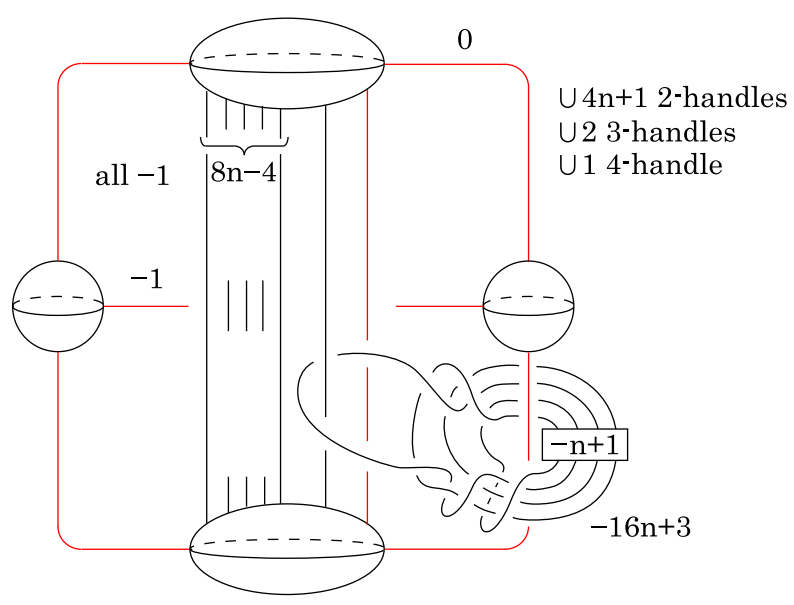

Figure $41 . E(n)_{4}$

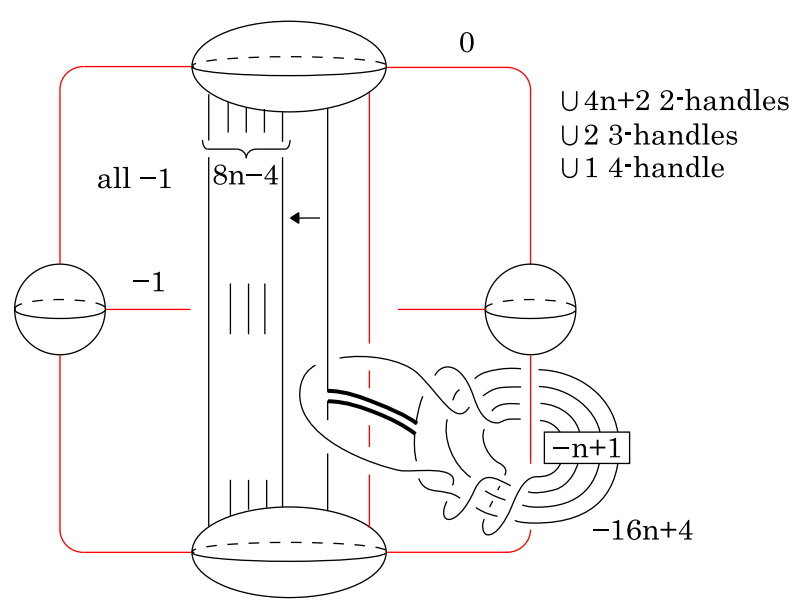

Figure $42 . \quad E(n)_{4}$

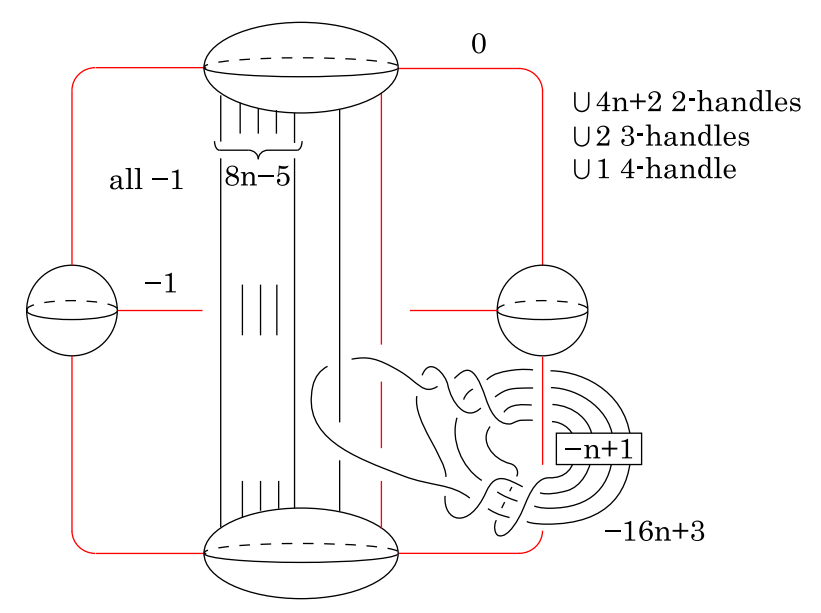

Figure 43. $E(n)_{4}$ 


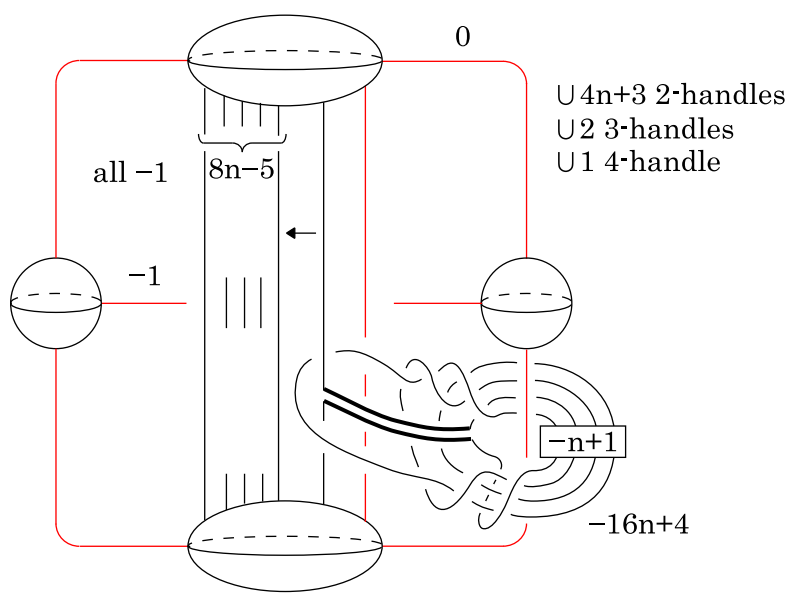

Figure $44 . E(n)_{4}$

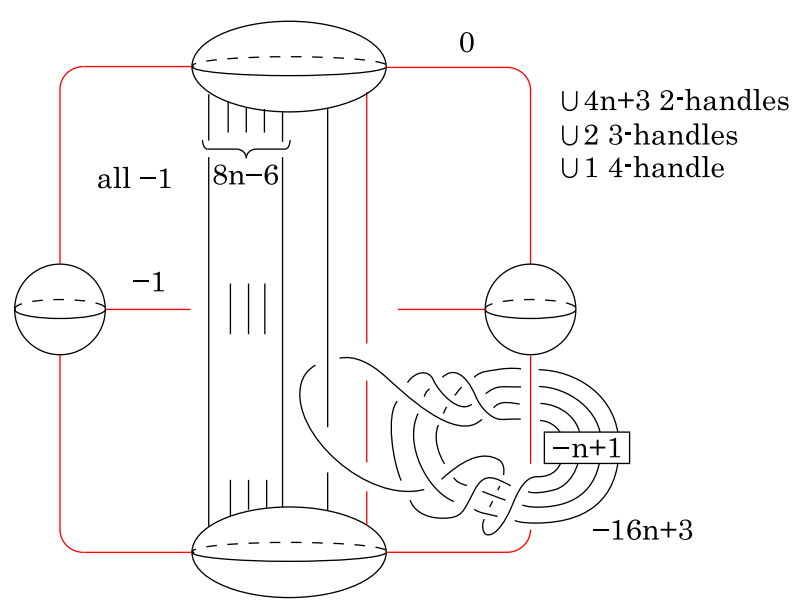

Figure $45 . \quad E(n)_{4}$

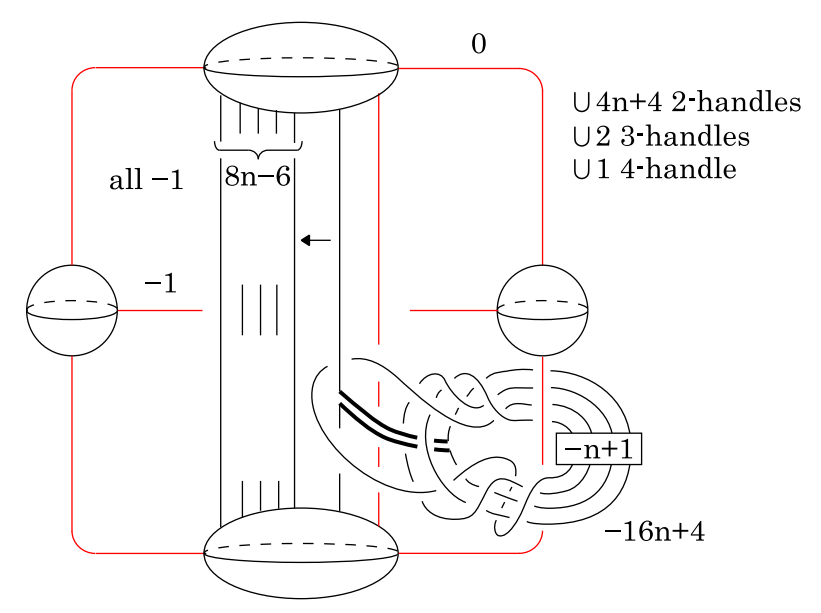

Figure 46. $E(n)_{4}$ 


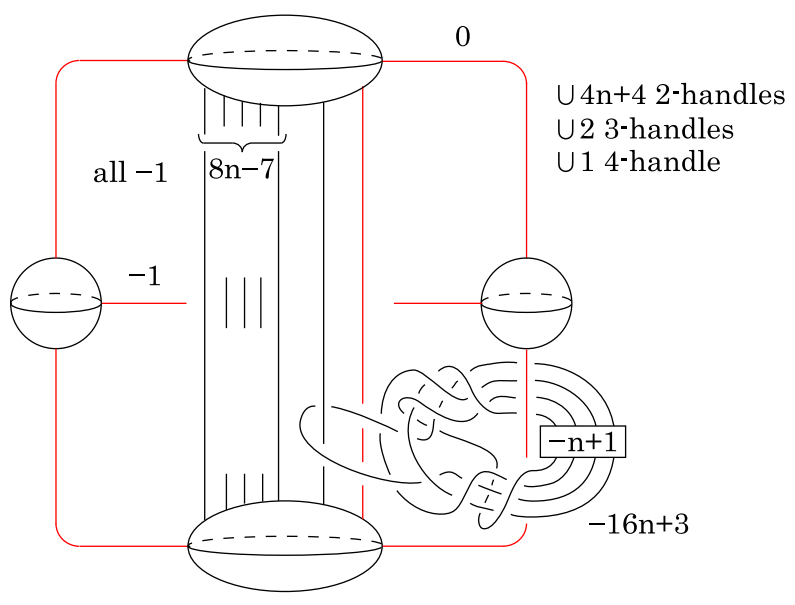

Figure $47 . E(n)_{4}$

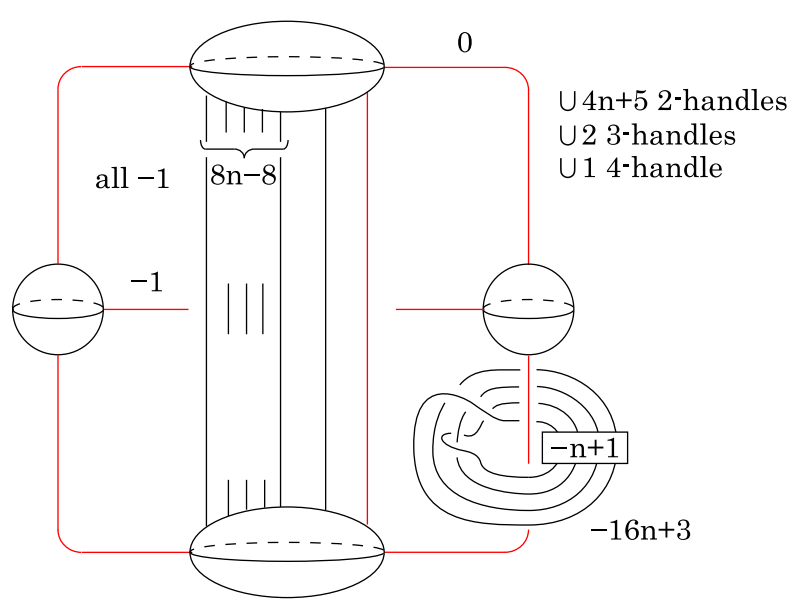

Figure $48 . \quad E(n)_{4}$

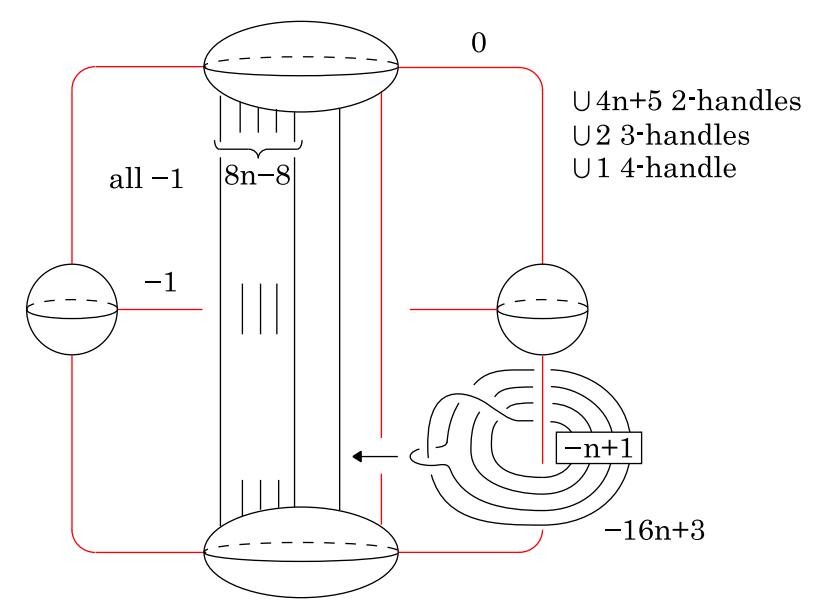

Figure 49. $E(n)_{4}$ 


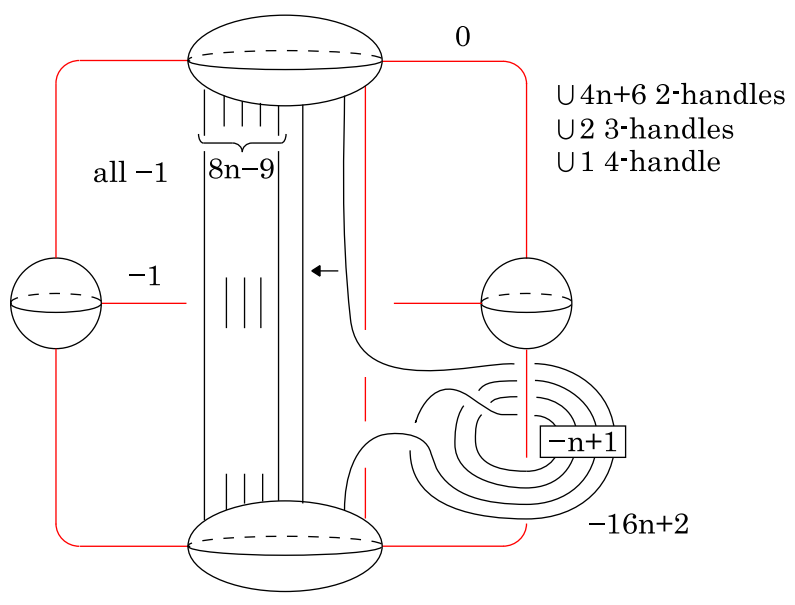

Figure 50. $E(n)_{4}(n \geq 2)$

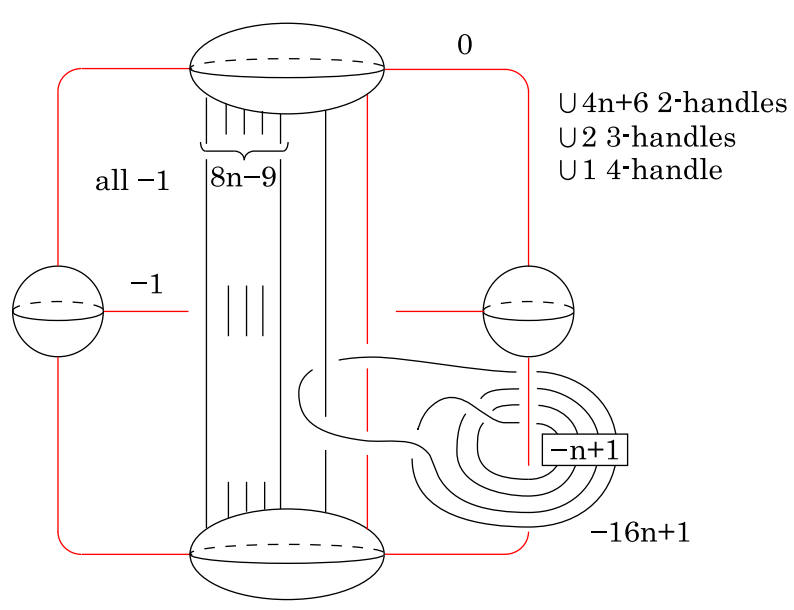

Figure 51. $E(n)_{4}(n \geq 2)$

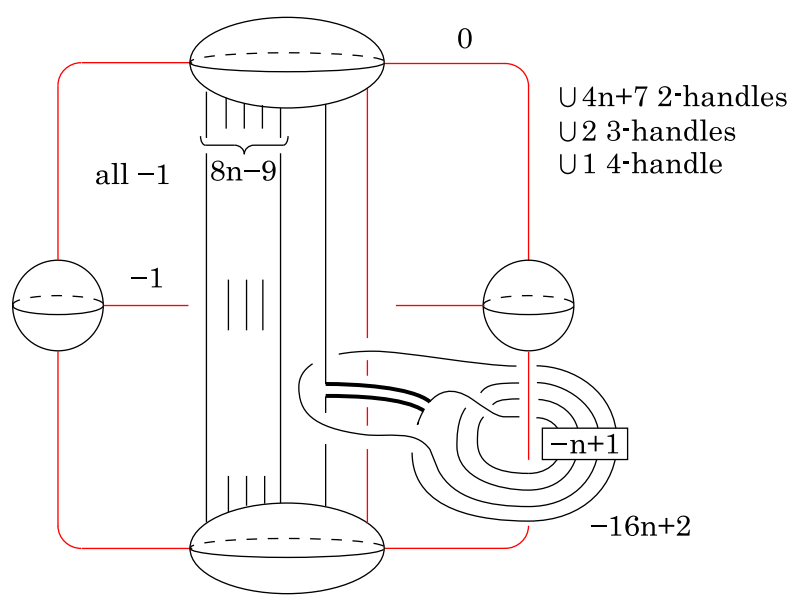

Figure $52 . E(n)_{4}(n \geq 2)$ 


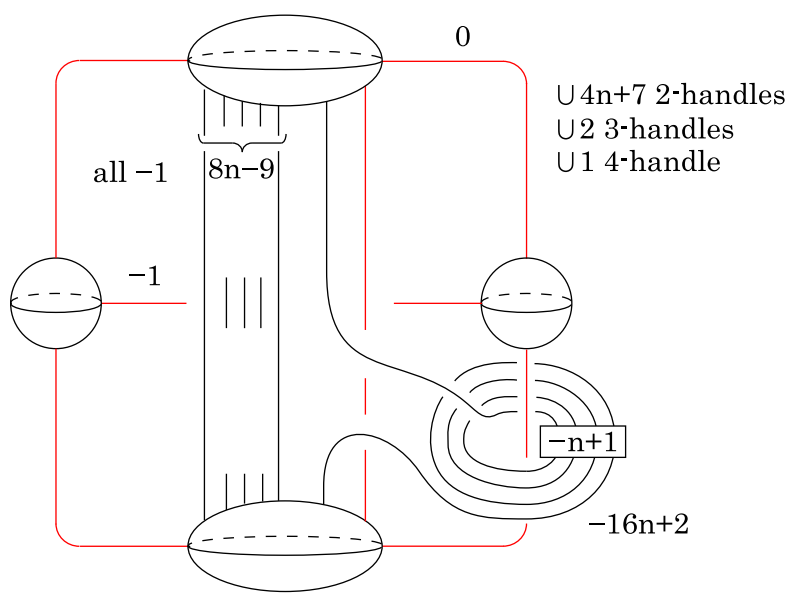

Figure 53. $E(n)_{4}(n \geq 2)$

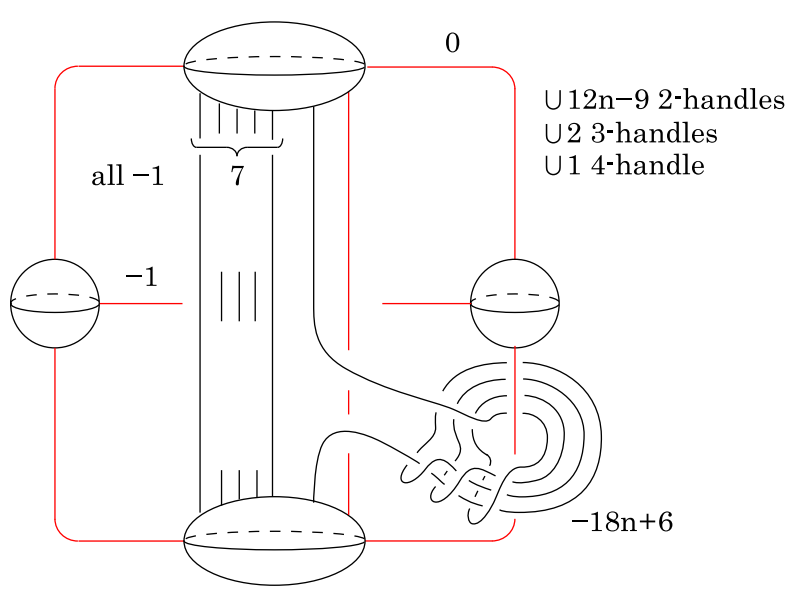

Figure 54. $E(n)_{4}$

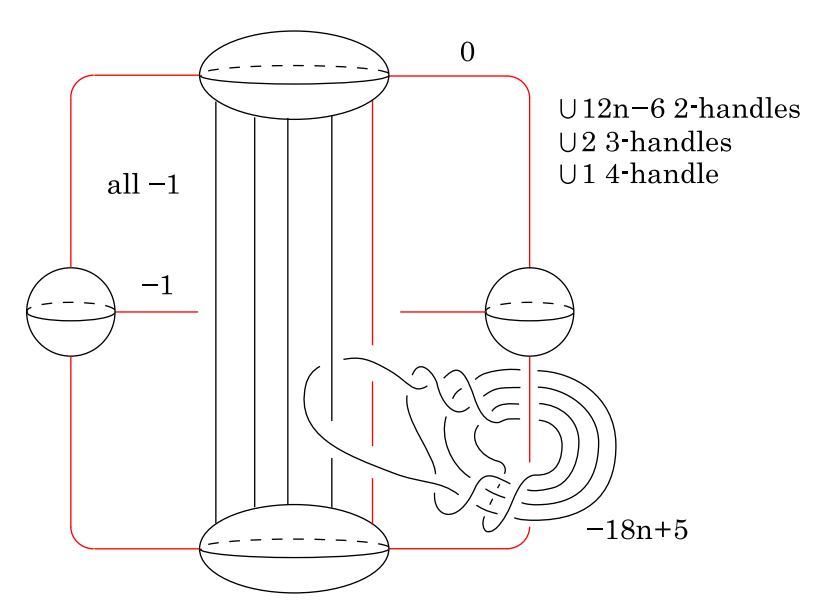

FiguRE 55. $E(n)_{4}$ 


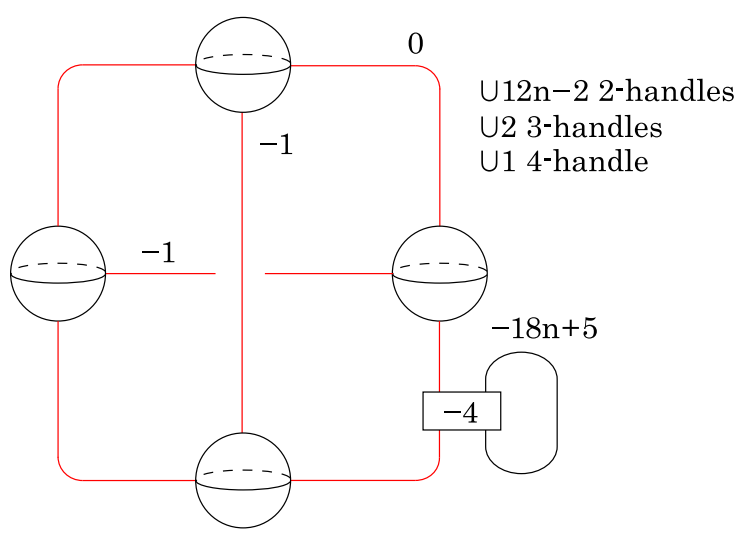

FiguRE $56 . \quad E(n)_{4}$ 